\title{
Single Image Haze Removal Algorithm using Color Attenuation Prior and Multi-Scale Fusion
}

\author{
Krati Katiyar \\ Trinity College of Engineering Bhopal, India
}

\author{
Neha Verma \\ Trinity College Engineering Bhopal, India
}

\begin{abstract}
This paper compares the Fast Single Image Haze Removal (FSIHR) using Color Attenuation Prior (CAP) and MultiScale Fusion (MSF) methods. Single image haze removal has been a challenging problem due to its ill-posed environment. FSIHR works as simple but powerful color attenuation earlier, for removal of haze from a single input hazy image. MSF method is a fusion-based approach that results from two original hazy image inputs by applying a white balance and a contrast enhancing process. To merge the information of the derived inputs successfully, to maintain the regions with good visibility, it filters their important features by computing three measures (weight maps): luminance (Y), chromaticity (C), and saliency (S). The other FSIHR using CAP creates a linear model for modeling the picture depth of the hazy image with a supervised learning method; the depth information can be well recovered. With the depth map of the hazy image, the transmission and the scene radiance restoration via the atmospheric scattering model, and thus efficiently remove the haze from a single image. While the MSF method is faster than existing single image dehazing strategies and yields precise results.
\end{abstract}

\section{Keywords}

Dehazing, image defogging, image restoration, depth estimation.

\section{INTRODUCTION}

Outdoor images taken in bad weather conditions (e.g., foggy or hazy) generally lose contrast and fidelity, resulting from the fact that light is absorbed and scattered by the cloudy medium such as particles and water droplets in the atmosphere through the process of propagation. Reinstatement of images taken in these specific situations has caught increasing attention in the last years. This job is important in a number of outdoor applications such as remote sensing, intelligent vehicles, object recognition and surveillance. In remote sensing systems, the recorded bands of reflected light are processed [1], [2] in order to restore the outputs. Multi-image techniques [3] solve the image de hazing difficulty by processing several input images that have been taken in different atmospheric situation. A more difficult problem is when only a single degraded image is available. Solutions for such cases have been introduced only in recent times [6]-[10]. In this paper we introduce an alternative single-image based approach that is able to accurately dehaze images using only the original degraded information. An extended conceptual of the core idea has been recently introduced by the authors in [11]. Moreover, most automatic systems, which strongly depend on the meaning of the input images, fail to work usually caused by the degraded images. Therefore, improved techniques of image haze removal will benefit many image understanding and computer vision applications such as aerial imagery [1], image classification [2]-[5],image/video retrieval [6]-[8], remote sensing [9]-[11] and video analysis and recognition
[12]-[14].Since concentration of the haze is different from place to place and it is difficult to detect in a hazy image, image dehazing is thus a challenging task. Although Tan's approach is able to attain notable results, it tends to produce over-saturated images. Fattal [15] proposes to remove the haze from color images based on Independent Component Analysis (ICA), but the approach is time-consuming and cannot be used for grayscale image dehazing.

First, this approach performs an effective per-pixel calculation, different from the majority of the earlier methods [6]-[8] that process patches. An appropriate per-pixel strategy reduces the amount of artifacts, since patch based methods have some limitations due to the assumption of constant air light in every patch. In broad, the assumptions made by patchbased techniques do not hold, and therefore additional post processing steps are necessary (e.g. the method of He et al. [8] needs to smooth the transmission map by alpha-matting). Secondly, since do not estimate the depth (transmission) map, the difficulty of this approach is lower than most of the earlier strategies. Finally, this technique performs faster which makes it appropriate for real-time applications.

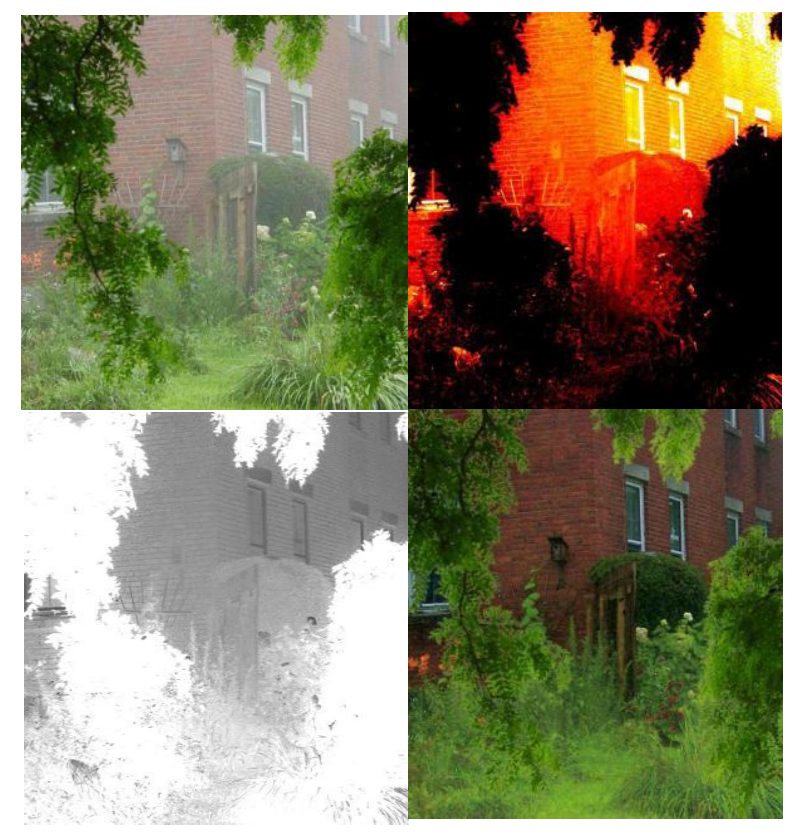

Fig. 1.An overview of the proposed dehazing method. Topleft: Input hazy image. Top-right: Restored depth map. Bottom-left: Restored transmission map. Bottom-right: Dehazed image.

Even compared with the recent effective implementation of Tarel and Hautière [10] this technique is able to restore a hazy image in less time, while screening more visually plausible results in terms of colors and details 
Furthermore, it has some complications to deal with densehaze images. Inspired by the widely used dark-object subtraction technique [16] and based on a large number of experiments on haze-free images, He et al. [17] discover the dark channel prior (DCP) that, in most of the non-sky patches, at least one color path has some pixels whose intensities are very low and close to zero. With this prior, they restore the haze-free image by the atmospheric scattering model and they estimate the thickness of haze. Figure 1 shows types of hazerelevant features with Random Forest [52] to estimate the transmission. Also overview of the CAP dehazing method is shown in Figure 1. The efficiency of this dehazing method is dramatically high and the dehazing efficacy is also superior to that of prevailing dehazing algorithms as we will show in Section 6. A conference version of our work has been presented in [53].The remainder of this paper is organized as follows:

In Section 2, we review the atmospheric scattering model which is widely used for image dehazing and give a concise analysis on the parameters of this model. In Section 3, we present a novel color attenuation prior. In Section 4, we discuss the approach of recovering the scene depth with the proposed color attenuation prior. In Section V, the method of image dehazing with the depth information is described. In Section 6, we present and analyze the experimental results. Finally, we summarize this paper in Section 7.

\section{ATMOSPHERIC SCATTERING MODEL}

To explain the configuration of a hazy image, the atmospheric scattering model, which is proposed by McCartney in 1976 [20], is generally used in computer vision and image processing. Narasimhan and Nayar [21], [22], [23], [24]further derive the model later, and the model can be expressed as follows:

$$
\begin{aligned}
& I(x)=J(x) t(x)+A(1-t(x)) \\
& t(x)=e^{-\beta d(x)}
\end{aligned}
$$

Where $x$ is the position of the pixel within the image, I is the hazy image, $\mathrm{J}$ is the scene radiance indicating the haze-free image, $\mathrm{A}$ is the atmospheric light, $t$ is the medium transmission, $\beta$ is the scattering coefficient of the atmosphere and $d$ is the depth of scene. I, $\mathbf{J}$ and Aare all 3-D vectors in RGB space. Since I is known, the objective of dehazing is to estimate $\mathrm{A}$ and $t$, then restore $\mathrm{J}$ according to Equation (1).It is significance noting that the depth of the scene $d$ is the most important information. Since the scattering coefficient $\beta$ can be regarded as a constant in homogeneous atmosphere condition [23], the average transmission $t$ can be approximate easily according to Equation (2) if the depth of the scene is known. Moreover, in the ideal case, the range of $d(x)$ is $[0$, $+\infty)$ as the scenery objects that show in the image can be very far away from the spectator, and we have:

$$
I(x)=A, d(x) \rightarrow \infty
$$

Equation (3) shows that the intensity of the pixel, which makes the depth tend to infinity, be able to stand for the value of the atmospheric light A. Note that, if $d(x)$ is large enough , $t$ $(x)$ tends to be very small according to Equation (2), and $\mathrm{I}(x)$ equals A approximately. Therefore, instead of calculating the atmospheric light $\mathrm{A}$ by Equation (3), we can approximation $\mathrm{A}$ by the following equation given a threshold $\mathrm{d}_{\text {thresold }}$ :

$$
I(x)=A, d(x) \geq d \text { thresold } .
$$

We also see the fact that it is not hard to satisfy this constraint: $d(x)>d_{\text {thresold }}$. In most cases, a hazy image taken outdoor has a far-away view that is kilometers away from the observer. In other words, the pixel belonging to the area with a far-away view in the image should have a very large depth $\mathrm{d}_{\text {thresold }}$. Assuming that every hazy image has a distantview, we have:

$$
d(x) \geq d_{\text {threshold }}, x \in\{x \mid \forall y: d(y) \leq d(x)\}
$$

Based on this hypothesis, the atmospheric light $\mathrm{A}$ is given by:

$$
A=I(x), x \in\{x \mid \forall y: d(y) \leq d(x)\} .
$$

On this condition, the job of dehazing can be further changed into depth information restoration. However, it is also a demanding task to obtain the depth map from a single hazy image. In the next part, present a novel color attenuation prior which is useful for restoring the depth information from a single hazy image directly.

Multi-Scale Fusion (MSF)
A. Input
B. Weighting map
C. MS fusion

In the fusion process, the inputs are weighted by specific computed maps in order to preserve the most significant detected quality. Each pixel $x$ of the output $F$ is computed by summing the inputs $I_{k}$ weighted by equivalent normalized weight $\operatorname{maps} W^{K}$ :

$$
F(x)=\Sigma_{k} W^{K}(x) I_{k}(x)
$$

where $I_{k}$ symbolizes the input ( $k$ is the index of the inputs) that is weighted by the normalized weight maps $W^{K}$.The normalization of the weights ensures that the intensity scale of the result is maintained in relatively the same scale as the inputs (since the sum of each pixel equals $1, \Sigma_{k} W^{K}(x)=1$ ). The youthful result (refer to figure 2 ) that directly implements this equation, introduces strong halos artifacts, mostly in the locations considered by strong transitions of the weight maps. To check such degradation problems, we have opted for the adapted solution that employs a classical multi-scale pyramidal modification strategy [25].It is also tested by several.
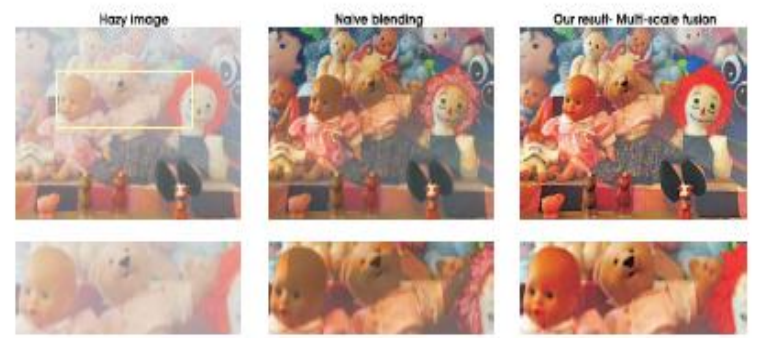

Fig. 2. The naive blending that directly implements equation 12 introduces halo artifacts, most apparent in locations characterized by strong transitions of the weight maps.

More recent edge preserving techniques (e.g. WLS [26]) but it cannot obtain significant improvement. However, recent superior methods need, in general, to pinch their parameters, as well as being more computationally concentrated. In this case, each $\operatorname{input} I_{k}$, is decomposed into a pyramid by applying Laplacian operator at different scales. Likewise, for each normalized weight map $W^{K}$, a Gaussian pyramid is computed. Considering that both the Gaussian and Laplacian pyramids have the same number of levels, the combination between the Laplacian inputs and Gaussian normalized 
weights is performed at each level independently, yielding the fused pyramid:

$$
F_{l}(x)=\Sigma_{k} G_{l}\left\{W^{K}(x)\right\} L_{l}\left\{I_{k}(x)\right\}
$$

where $l$ represents the number of the pyramid levels (default value of the number of levels is $l=5$ ) and $L\{I\}$ is the Laplacian account of the input $I$ while $G\{W\}$ represents the Gaussian account of the normalized weight map of the $W$. This step is performed sequentially for each pyramid layer, in a bottom-up manner. The final haze-free image $J$ is obtained by summing the contribution of the resulting inputs (levels of pyramid):

$$
J(x)=\Sigma_{l} F_{l}(x) \uparrow^{d}
$$

where $\uparrow^{d}$ is the upsampling operator with factor $d=2^{l-1}$. As a default characteristic, in this execution the contribution of all the three weight maps is equally distributed.

\section{COLOR ATTENUATION PRIOR}

As very small information about the scene structure is available it is very difficult to detect or remove the haze from a distinct image in computer vision, In spite of this, the human brain can quickly recognize the hazy area from the natural scenery without any extra information. This motivated us to conduct a large number of experiments on a variety of hazy images to find the information and seek a new prior for single image dehazing. Interestingly, find that the brightness and the saturation of pixels in a hazy image vary sharply along with the change of the haze attention. It seems that the three properties (the brightness, the saturation and the difference) are prone to vary frequently in a sole hazy image according to this observation. Is this coincidence, or is there a fundamental reason behind this? To find the answer this question, first re-examine the process of imaging. In the hazefree condition, the scene element reflects the energy that is from the lighting source (e.g., direct sunlight, diffuse skylight and light reflected by the ground), a portion of energy is lost when it reaches the imaging system. The imaging system collects the inward energy reflected from the scene part and focuses it onto the image plane. Without the effect of haze, outdoor images are typically are of different colors. In hazy conditions, in contrast, the situation becomes more difficult. There are two mechanisms (the direct attenuation and the air light) in imaging under hazy conditions [22]. On one hand, the direct attenuation caused by the decrease in reflected energy leads to low intensity of the brightness. To recognize this, review the atmospheric scattering method. The term $\mathrm{J}(x) t(x)$ in Equation (1) is used for describing the direct attenuation. It reveals the fact that the intensity of the pixels within the image will reduce in a multiplicative way. So it turns out that the brightness tends to decrease under the control of the direct attenuation. On the other hand, the white or gray air light, which is produced by the scattering of the environmental illumination, enhances the brightness and minimizes the saturation. This can also be explained by the atmospheric scatter model. The rightmost term A $(1-t(x))$ in Equation (1) represents the effect of the air light. It can be deduced from this term that the effect of the white or gray air light on the experiential values is additive. Thus, caused by the air light, the brightness is increased while the saturation is decreased. Since the air light plays a more important role in most cases, hazy areas in the image are categorized by high brightness and low saturation. The denser the haze is, the stronger the influence of the air light would be. Since the concentration of the haze increases along with the change of the scene depth in general, make an assumption that the depth of the scene is positively correlated with the concentration of the haze and it gives:

$$
D(x) \propto c(x) \propto v(x)-s(x),
$$

Where $d$ is the scene depth, $c$ is the concentration of the haze, $v$ is the brightness of the scene and $s$ is the saturation. We observe this statistics as color attenuation prior. Figure 2gives the statistical explanation of the color attenuation prior through the HSV color model.

\section{SCENE DEPTH RESTORATION 4.1 The Linear Model Definition}

As the disparity between the brightness and the saturation can approximately represent the concentration of the haze, then it can create a linear model, i.e., a more precise expression, as follows:

$$
d(x)=\theta_{0}+\theta_{1} v(x)+\theta_{0} s(x)+\varepsilon(x),(11)
$$

Where $x$ is the position within the image, $d$ is the scene depth, $v$ is the brightness component of the hazy image, $s$ is the saturation component, $\theta 0, \theta 1, \theta 2$ are the unknown linear coefficients, $\varepsilon(x)$ is a random variable expressive the random error of the model, and $\varepsilon$ can be regarded as a random image. Use a Gaussian density for $\varepsilon$ with zero mean and variable $\sigma 2$ (i.e. $\varepsilon(x) \sim N(0, \sigma 2)$ ). According to the property of the Gaussian distribution:

$$
d(x) \sim p\left(d(x) \mid x, \theta_{0}, \theta_{1}, \theta_{2}, \sigma^{2}\right)=N\left(\theta_{0}+\theta_{1} v+\theta_{2} s, \sigma^{2}\right)
$$

One of the most important advantages of this model is that it has the edge-preserving property. To show this, calculate the gradient of $d$ in Equation (8):

$$
\nabla d=\theta_{1} \nabla v+\theta_{2} \nabla s+\nabla \varepsilon
$$

Due to that $\sigma$ can never be too large in practice; the value of $\varepsilon(x)$ tends to be very low and close to zero. In this case, the value of $\nabla \varepsilon$ is low enough to be ignored.

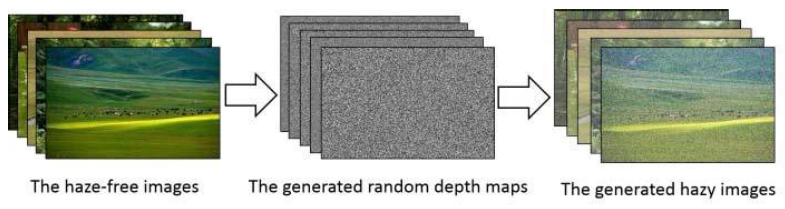

Fig. 3. The process of generating the training samples with the hazefree images. Left sub-figure: The haze-free images. Center sub-figure: The generated random depth maps. Right sub-figure: The generated hazy images.

This further ensures that the depth information can be well recovered even near the depth discontinuities in the scene. In the following sections, use a simple and efficient supervised learning method to determine the coefficients $\theta_{0}, \theta_{1}, \theta_{2}$ and the variable $\sigma^{2}$

\subsection{Training Data Collection}

In order to discover the coefficients $\theta 0, \theta 1$ and $\theta 2$ exactly, the training data are needed. In this case, a training sample consists of a hazy image and its corresponding ground fact depth map. Unfortunately, the depth map is very complex to obtain due to the fact that there is no reliable means to calculate the depths in outdoor scenes. Current depth cameras such as Kinect are not able to obtain the accurate depth information. Inspired by Tang et al.'s method for preparing the training data [27], then collect the haze-free images from Google Images and Flickr and use them to create the synthetic depth maps and the equivalent hazy images for obtaining sufficient training samples. 
C. Learning Strategy

In the joint conditional concentration:

$$
L=p\left(d\left(x_{1}\right), \ldots, d\left(x_{n}\right) \mid x_{1}, \ldots, x_{n}, \theta_{0}, \theta_{1}, \theta_{2}, \sigma^{2}\right)
$$

wheren is the total number of pixels within the training hazy images, $d(x n)$ is the depth of the nth scene point, and $L$ is the likelihood. Let that the random error at each scene point is

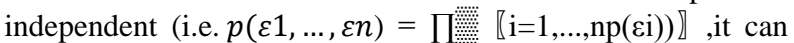
rewrite Equation (11) as:

$$
\left.L=\prod_{i=1}^{n} p d\left(x_{i}\right) \mid x_{i}, \theta_{0}, \theta_{1}, \theta_{2}, \sigma^{2}\right)
$$

According to Equation (9) and Equation (12), it gives:

$$
L=\prod_{i=1}^{n} \frac{1}{\sqrt{2 \pi \sigma^{2}}} e^{-\frac{d g_{i}-\left(\theta_{0}+\theta_{1} v\left(x_{i}\right)+\theta_{2} s\left(x_{i}\right)\right)}{2 \sigma^{2}}}
$$

where $d g_{i}$ represents the ground truth depth of the ith scene point. So the problem is to find the optimal values of $\theta 0, \theta 1$, $\theta 2$, and $\sigma$ to maximum $L$. For convenience, instead of maximizing the probability directly, we maximize the natural $\operatorname{logarithm}$ of the probability $\ln L$. Therefore, the problem can be expressed as follows:

$\operatorname{argmax}_{\theta_{0}, \theta_{1}, \theta_{2}, \sigma} \ln L=\sum_{i=1}^{n} \ln \left(\frac{1}{\sqrt{2 \pi \sigma^{2}}} e^{-\frac{d g_{i}-\left(\theta_{0}+\theta_{1} v\left(x_{i}\right)+\theta_{2} s\left(x_{i}\right)\right)}{2 \sigma^{2}}}\right)$

To solve the problem, first calculate the partial derivative of $\ln L$ with respect to $\sigma$ and make it equal to zero:

$$
\frac{\partial \ln L}{\partial \sigma}=\frac{n}{\sigma}+\frac{1}{\sigma^{3}} \sum_{i=1}^{n}\left(d g_{i}-\left(\theta_{0}+\theta_{1} v\left(x_{i}\right)+\theta_{2} s\left(x_{i}\right)\right)\right)=0
$$

According to Equation (18), the maximum likelihood estimate for the variable $\sigma 2$ is:

$$
\sigma^{2}=\sum_{i=1}^{n}\left(d g_{i}-\left(\theta_{0}+\theta_{1} v\left(x_{i}\right)+\theta_{2} s\left(x_{i}\right)\right)\right)^{2}
$$

As for the linear coefficients $\theta 0, \theta 1$ and $\theta 2$ use the gradient descent algorithm to estimate their values. By taking the partial derivatives of $\ln L$ with respect to $\theta 0, \theta 1$ and $\theta 2$ respectively,

Algorithm 1 Parameters Estimation

Input the training brightness vector $\mathrm{v}$, the training saturation vector $\mathrm{s}$, the training depth vector $\mathrm{d}$, and the number of iterations $t$

Output linear coefficient $\theta_{0}, \theta_{1}, \theta_{2}$, the variance $\sigma^{2}$

Auxiliary functions:

Function for obtaining the size of the vector: $n=s i z e$ (in)

Function for calculating the square: out=square (in)

\section{Begin}

$1: \mathrm{n}=\operatorname{size}(\mathrm{v})$

2: $\theta_{0}=0 ; \theta_{1}=1 ; \theta_{2}=-1$;

$3:$ sum $=0 ; w$ Sum $=0 ;$ vSum $=0 ;$ sSum $=0$;

4: for iteration from 1 to $t$ do

5: for index from 1 to $\mathrm{n}$ do

6: $\quad$ temp $=d[i]-\theta_{0}-\theta_{1} * v[i]-\theta_{2} * s[i]$;

7: $\quad$ wSum $=$ vSum + temp;

8: $\quad$ sSum $=s$ Sum $+v[i] *$ temp;

9: $\quad$ sSum $=$ sSum $+s[i] *$ temp;

10: $\quad$ sum $=$ sum + square $($ temp $)$;

11: $\quad$ end for

12: $\quad \sigma^{2}=\operatorname{sum} / n$;

13: $\quad \theta_{0}=\theta_{0}+w$ Sum $; \theta_{1}=\theta_{1}$ vSum; $\theta_{2}=\theta_{2}+$ sSum

14: $\quad$ end for

End

It can obtain the following expressions:

$$
\begin{gathered}
\frac{\partial \ln L}{\partial \theta_{0}}=\frac{1}{\sigma^{2}} \sum_{i=1}^{n}\left(d g_{i}-\left(\theta_{0}+\theta_{1} v\left(x_{i}\right)+\theta_{2} s\left(x_{i}\right)\right)\right) \\
\frac{\partial \ln L}{\partial \theta_{1}}=\frac{1}{\sigma^{2}} \sum_{i=1}^{n} v\left(x_{i}\right)\left(d g_{i}-\left(\theta_{0}+\theta_{1} v\left(x_{i}\right)+\theta_{2} s\left(x_{i}\right)\right)\right) \\
\frac{\partial \ln L}{\partial \theta_{2}}=\frac{1}{\sigma^{2}} \sum_{i=1}^{n} s\left(x_{i}\right)\left(d g_{i}-\left(\theta_{0}+\theta_{1} v\left(x_{i}\right)+\theta_{2} s\left(x_{i}\right)\right)\right)
\end{gathered}
$$

The expression for updating the linear coefficients can be in brief expressed by:

$$
\theta_{i}=\theta_{i}+\frac{\partial \ln L}{\partial \theta_{i}} \text { s.t. } i \in\{0,1,2\}
$$

It is worth noting that the expression above is used for iterating dynamically, and the notation: $=$ does not express the mathematical equality, but means that setting the value of $\theta \mathrm{i}$ in the left term to be the value of the right term. The process for learning the linear coefficients $\theta 0, \theta 1, \theta 2$ and the variable $\sigma 2$ is shown in Algorithm 1.

\subsection{Estimation of the Depth Information}

As the link among the scene depth $d$, the brightness $v$ and the saturation $s$ has been recognized and the coefficients have been estimated, then it can restore the depth map of a given input hazy image according to Equation (8).However, this model may fail to work in some particular situations. For example, the white objects in an image are frequently with high values of the brightness and low values of the saturation. Therefore, the model tends to consider the scene objects with white color as being far-away. Unluckily, this misclassification will result in inaccurate estimation of the depth in some cases. In the white geesein the image are the regions for which the model can barely handle, and these regions are wrongly estimated with high depth values in the depth map. To overcome this problem, need to consider each pixel in the neighborhood. Based on the hypothesis that the scene depth is locally constant, and then process the raw depth map by:

$$
d_{r}(x)=\min \min _{y \in \Omega_{r}(x)} d(y)
$$

Where $r(x)$ is an $r \times r$ neighborhood centered at $x$, and $d r$ is the depth map with scale $r$. The new depth map $d 15$ can well handle the geese regions. However, it is also obvious that the blocking artifacts show in the image. To process the depth map, use the guided image filtering [28]to smooth the image. The final restored depth map of the hazy image can be seen, the blocking artifacts.

\section{SCENE RADIANCE RECOVERY}

\subsection{Estimation of the Atmospheric Light}

The main idea of estimating the atmospheric light explained in Section II. In this section, explain the method in more detail. As the depth map of the input hazy image has been improved, the allocation of the scene depth is known. Bright regions in the map stand for distant places. According to Equation (6), pick the top0.1 percent brightest pixels in the depth map, and select the pixel with maximum intensity in the corresponding hazy image I among these brightest pixels as the atmospheric light $\mathrm{A}$

\subsection{Scene Radiance Recovery}

Now that the depth of the scene $d$ and the atmospheric light Aare known, it can estimate the medium transmission $t$ simply according to Equation (2) and improve the scene radiance Jin Equation (1). For ease, rewrite Equation (1) as follows:

$$
J(x)=\frac{I(x)-A}{t(x)}+A=\frac{I(x)-A}{e^{-\beta d(x)}}+A
$$


For avoiding producing too much noise, limit the value of the transmission $t(x)$ between 0.1 and 0.9 . So the final function used for restoring the scene radiance $\mathbf{J}$ in the method can be expressed by:

$$
J(x)=\frac{I(x)-A}{\min \left\{\max \left\{e^{-\beta d(x)}, 0.1\right\}, 0.9\right\}}+A
$$

where Jis the haze-free image. Figures (4) show some finishing results of dehazing of the given method. Note that the scattering coefficient $\beta$, which can be regarded as a constant [23] in homogeneous regions, the ability of a unit volume of atmosphere to scatter light in all directions. In other words, $\beta$ determines the intensity of dehazing indirectly. Therefore, a moderate $\beta$ is essential when dealing with the images with dense-haze regions. In most cases, $\beta=1.0$ is more than enough.

\section{EXPERIMENTS}

In order to verify the usefulness of the given dehazing method, test it on various hazy images and compare with $\mathrm{He}$ et al.'s [17] method. All the algorithms are implemented in the MatlabR2012a environment on a P4-2.3GHz PC with 4GB RAM. The parameters used in the given method are initialized as follows: $r=15, \beta=1.0, \theta 0=0.121779$,

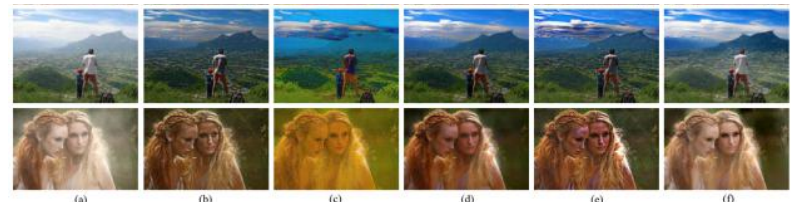

Fig. 4. Qualitative comparison of different methods on real-world images (a) The hazy images (b) Tarel et al.'s results (c) Nishino et al.'s results(d) He et al.'s results (e) Meng et al.'s results (f) multiscale methods result

\section{DISCUSSIONS AND CONCLUSION}

In this work the linear CAP based dehazing method creates a linear model for the scene depth of the hazy image. The supervised learning method is used for the parameter learning of this model through which the depth information can be well recovered. By means of the depth map obtained by the given method, the scene radiance of the hazy image can be recovered effortlessly. In a fusion-based approach, the first fusion based strategy that is able to resolve fog and haze problems using only one degraded image. By choosing appropriate weight maps and inputs, a multi-scale fusion strategy can be used to efficiently dehaze images. The MSF method is faster than existing single image dehazing strategies and yields precise results. In future work these methods may be tested on videos.

\section{REFERENCES}

[1] G. A. Woodell, D. J. Jobson, Z.-U. Rahman, and G. Hines, "Advanced image processing of aerial imagery," Proc. SPIE, vol. 6246, p. 62460E, May 2006.

[2] L. Shao, L. Liu, and X. Li, "Feature learning for image classification viamulti objective genetic programming," IEEE Trans. Neural Netw. Learn.Syst., vol. 25, no. 7, pp. 1359-1371, Jul. 2014.

[3] F. Zhu and L. Shao, "Weakly-supervised cross-domain dictionary learning for visual recognition," Int. J. Comput. Vis., vol. 109, nos. 1-2,pp. 42-59, Aug. 2014.

[4] Y. Luo, T. Liu, D. Tao, and C. Xu, "Decompositionbased transfer distance metric learning for image classification," IEEE Trans. ImageProcess., vol. 23, no. 9, pp. 3789-3801, Sep. 2014.

[5] Tao, X. Li, X. Wu, and S. J. Maybank, "Geometric mean for subspaceselection," IEEE Trans. Pattern Anal. Mach. Intell., vol. 31, no. 2,pp. 260-274, Feb. 2009.

[6] J. Han et al., "Representing and retrieving video shots in human-centricbrain imaging space," IEEE Trans. Image Process., vol. 22, no. 7,pp. 2723-2736, Jul. 2013.

[7] J. Han, K. Ngan, M. Li, and H.-J. Zhang, "A memory learning frameworkfor effective image retrieval," IEEE Trans. Image Process., vol. 14,no. 4, pp. 511-524, Apr. 2005.

[8] Tao, X. Tang, X. Li, and X. Wu, "Asymmetric bagging and randomsubspace for support vector machines-based relevance feedback in imageretrieval," IEEE Trans. Pattern Anal. Mach. Intell., vol. 28, no. 7,pp. 1088-1099, Jul. 2006.

[9] J. Han, D. Zhang, G. Cheng, L. Guo, and J. Ren, “Object detection inoptical remote sensing images based on weakly supervised learning andhigh-level feature learning," IEEE Trans. Geosci. Remote Sens., vol. 53,no. 6, pp. 3325-3337, Jun. 2015.

[10] Cheng et al., "Object detection in remote sensing imagery using adiscriminatively trained mixture model," ISPRS J. Photogramm. RemoteSens., vol. 85, pp. 32-43, Nov. 2013.

[11] J. Han et al., "Efficient, simultaneous detection of multiclass geospatialtargets based on visual saliency modeling and discriminative learning ofsparse coding," ISPRS $J$. Photogramm. Remote Sens., vol. 89, pp. 37-48, Mar. 2014.

[12] L. Liu and L. Shao, "Learning discriminative representations fromRGB-D video data," in Proc. Int. Joint Conf. Artif. Intell., Beijing, China,2013, pp. 1493 1500 .

[13] D. Tao, X. Li, X. Wu, and S. J. Maybank, "General tensor discriminant analysis and Gabor features for gait recognition," IEEE Trans. PatternAnal. Mach. Intell., vol. 29, no. 10, pp. 1700-1715, Oct. 2007.

[14] Z. Zhang and D. Tao, "Slow feature analysis for human actionrecognition," IEEE Trans. Pattern Anal. Mach. Intell., vol. 34, no. 3,pp. 436-450, Mar. 2012.

[15] R. Fattal, "Single image dehazing," ACM Trans. Graph., vol. 27, no. 3, p. 72, Aug. 2008.

[16] P. S. Chavez, Jr., "An improved dark-object subtraction technique for atmospheric scattering correction of multispectral data," Remote Sens. Environ., vol. 24, no. 3, pp. 459-479, Apr. 1988.

[17] K. He, J. Sun, and X. Tang, "Single image haze removal using dark channel prior," IEEE Trans. Pattern Anal. Mach. Intell., vol. 33, no. 12, pp. 2341-2353, Dec. 2011.

[18] L. Breiman, "Random forests," Mach. Learn., vol. 45, no. 1, pp. 5-32, Oct. 2001.

[19] Q. Zhu, J. Mai, and L. Shao, "Single image dehazing using color attenuation prior," in Proc. Brit. Mach. Vis. Conf. (BMVC), Nottingham, U.K., 2014, pp. 1-10. 
[20] E. J. McCartney, Optics of the Atmosphere: Scattering by Molecules and Particles. New York, NY, USA: Wiley, 1976.

[21] S. K. Nayar and S. G. Narasimhan, "Vision in bad weather," in Proc. IEEE Int. Conf. Comput. Vis. (ICCV), vol. 2. Sep. 1999, pp. 820-827.

[22] S. G. Narasimhan and S. K. Nayar, "Contrast restoration of weather degraded images," IEEE Trans. Pattern Anal. Mach. Intell., vol. 25, no. 6, pp. 713-724, Jun. 2003.

[23] S. G. Narasimhan and S. K. Nayar, "Vision and the atmosphere," Int. J. Comput. Vis., vol. 48, no. 3, pp. 233-254, Jul. 2002.

[24] S. G. Narasimhan and S. K. Nayar, "Removing weather effects from monochrome images," in Proc. IEEE Conf. Comput. Vis. PatternRecognit. (CVPR), 2001, pp. II186-II-193.

[25] J.-P. Tarel, N. Hautière, L. Caraffa, A. Cord, H. Halmaoui, and D. Gruyer, "Vision enhancement in homogeneous and heterogeneousfog," IEEE Intell. Transp. Syst. Mag., vol. 4, no. 2, pp. 6-20, Apr. 2012.

[26] C. O. Ancuti, C. Ancuti, C. Hermans, and P. Bekaert, "A fast semiinverse approach to detect and remove the haze from a single image," in Proc. Asian Conf. Comput. Vis. (ACCV), 2010, pp. 501-514

[27] K. Tang, J. Yang, and J. Wang, "Investigating hazerelevant features in a learning framework for image dehazing," in Proc. IEEE Conf. Comput. Vis. Pattern Recognit. (CVPR), Jun. 2014, pp. 2995-3002.

[28] K. He, J. Sun, and X. Tang, "Guided image filtering," IEEE Trans. Pattern Anal. Mach. Intell., vol. 35, no. 6, pp. 1397-1409, Jun. 2013

[29] A. J. Preetham, P. Shirley, and B. Smits, "A practical analytic model for daylight," in Proc. ACM Special Interest Group Comput.Graph. (SIGGRAPH), 1999, pp. $91-100$ 